\title{
MÉTODO DFT PARA PRODUÇÃO DE TOMATE EM AMBIENTE PROTEGIDO
}

\author{
DFT method for tomato production in uhheated greenhouse
}

\author{
Paulo Cezar Rezende Fontes ${ }^{1}$, José Márcio Oliveira Ribeiro ${ }^{2}$, Gildo Heringer Silva ${ }^{3}$
}

\begin{abstract}
RESUMO
Em ambiente protegido, os gastos com sementes de cultivares híbridas e fita gotejadora influenciam, acentuadamente, o custo de produção de tomate (Lycopersicon esculentum Mill.). Para reduzir custos, idealizou-se o método denominado DFT que combina a utilização de menores densidades de plantio (D), comprimento da fita gotejadora (F) e maior número de caules por planta, especialmente tutorados (T) com fitilho. Com o presente trabalho, objetivou-se comparar os métodos DFT com o convencional (TEST) de produção de tomate em ambiente protegido. Foram realizados dois experimentos, utilizando-se o híbrido Carmen. Os tratamentos foram instalados no delineamento inteiramente casualizado, com 12 e 6 repetições nos tratamentos TEST e DFT, respectivamente, sempre circundadas por bordaduras. A produção de fruto comercial (PFC), expressa em $\mathrm{kg} \mathrm{m}^{-2}$, verificada na TEST foi semelhante (exp.1) ou maior (exp.2) do que em DFT. Quando expressada em $\mathrm{kg}_{\text {planta }}{ }^{-1} \mathrm{e} \mathrm{kg} \mathrm{R}^{-1}$ gasto com material de consumo, a PFC foi maior no sistema DFT. O método DFT proposto pode ser utilizado na produção de tomate em ambiente protegido.
\end{abstract}

Termos para indexação: Lycopersicon esculentum, densidade de plantio, fita gotejadora, semente, híbrido, desbrota, tutoramento.

\section{ABSTRACT}

The expenditure with seed and drip tape has remarkable influence in tomato (Lycopersicon esculentum Mill.) production cost in greenhouse. To reduce the cost, the denominated DFT method was idealized, which combines smaller planting density (D), smaller drip tape length (F) and larger stem number left in plant, especially supported (T) by plastic string attached to a trellis. The present work aimed at comparing the DFT technique with the conventional method (TEST). Two experiments were accomplished with Carmen hybrid. Treatments were laid in completely randomized design with 12 and 6 tomato plants in TEST and DFT respectively, always surrounded by border plants. Expressed as $\mathrm{kg} \mathrm{m}^{-2}$, the commercial fruit yield (PFC) in the conventional method was similar (exp.1) and larger (exp.2) than in DFT. Expressed as $\mathrm{kg} \mathrm{plant}^{-1}$ and $\mathrm{kg} \mathrm{R}^{-1}$ expended in materials, the PFC was higher in DFT system. The proposed method can be utilized in tomato production in unheated greenhouse.

Index terms: Lycopersicon esculentum, planting density, drip tape, seed, hybrid, pruning, supporting.

(Recebido em 3 de Janeiro de 2005 e aprovado em 20 de março de 2006)

\section{INTRODUÇÃO}

O crescimento do tomateiro (Lycopersicon esculentum Mill.) depende da habilidade do aparato vegetativo em manter a sua capacidade de suprir os órgãos da planta, especialmente os frutos (GARY et al., 2003). Esses são integrantes fundamentais no mecanismo de retroalimentação do crescimento do tomateiro (HEUVELINK, 1996). Em grande parte, a produção de frutos é modulada pela combinação da densidade de plantio e número de caules por planta (CAMARGOS, 1998) a qual determina a área foliar disponível para a interceptação da radiação solar e realização de fotossíntese.

A máxima captação da luz por maior número de tomateiros (estande) e/ou de caule por unidade de área (fonte máxima) deve ser a meta do tomaticultor. A otimização das mesmas é fundamental para maximizar a produção de frutos do tamanho desejado. Assim, o "tamanho ideal da fonte" a ser deixada no tomateiro, via poda e desbrotas, depende do tamanho do dreno representado pelos números de cachos e de frutos.
Com o aumento da densidade de plantio, até determinado valor, a produção total de frutos por unidade de área se eleva (CAMPOS et al., 1987), ocorrendo, porém, decréscimo no peso médio e na produção por planta. Para a produção de frutos para mesa, no campo, a densidade de plantio do tomateiro tem variado de 1,40 a 3,30 plantas $\mathrm{m}^{-2}$ determinada pelo espaçamento entre fileiras de 1,0 a 1,2 m e entre plantas de 0,3 a 0,6 m, sendo mais comum 1,0 x 0,6 m (FONTES \& SILVA, 2002). Em casa-de-vegetação, a densidade varia de 1,66 a 5,30 plantas $\mathrm{m}^{-2}$ (CAMARGOS et al., 2002; CRUZ-CARRILLO et al., 2003).

Em cada tomateiro desenvolvem-se dezenas de caules. Pelas desbrotas ou podas freqüentes da gema axilar é comum deixar 1 ou 2 caules em cada planta. Por ser perene, em cada caule desenvolvem-se diversos cachos ao longo do ciclo da cultura. Quanto mais o caule alongase, maior é o número de cachos na planta. Mas, a altura do caule precisa ser compatível com a altura do tutor. Assim, atingida a altura desejada, a gema apical é eliminada, limitando-se o crescimento do caule e determinando-se o número de cachos que permanecem na planta.

\footnotetext{
'Professor Titular, PhD, UFV - DFT - 36570-000 - Viçosa, MG - Bolsista do CNPq - Apoio da FAPEMIG - pacerefo@ufv.br

${ }^{2}$ Engenheiro Agrônomo, MS, EPAMIG-CRPZM - 36.570-000 - Viçosa, MG - Ex-Bolsista do CNPq.

${ }^{3}$ Engenheiro Agrônomo - Rua Laranja da Terra - Bairro Rural - 29.390-000 - Luna, ES - Ex-Bolsista de Iniciação Científica do CNPq.
} 
Talvez, a combinação de baixa densidade de plantio e elevado número de caules por planta poderá resultar em produtividade e tamanho de frutos similares às obtidas com a combinação de alta densidade de plantio e menor número de caules por tomateiro, principalmente em ambiente protegido. Mas, a baixa relação fonte/dreno pode limitar o crescimento do fruto que são os maiores drenos. Assim, é necessário haver equilíbrio entre o carregamento de frutos e a parte vegetativa pois, ambos competem por fotoassimilados.

Em ambiente protegido, principalmente nos meses de dias longos e de intensa energia fotossinteticamente ativa, é pouco provável que o suprimento de fotoassimilados limite o desenvolvimento do tomateiro (GAUTIER et al., 2001). Na cultura do tomateiro em ambiente protegido, é comum a combinação do uso de híbrido e de irrigação por gotejamento as quais começam, também, a serem usadas no campo. Normalmente, a planta híbrida de tomate em ambiente protegido é plantada no espaçamento de $1 \mathrm{~m}$ entre fileira e $0,60 \mathrm{~m}$ entre plantas sendo conduzida com dois caules. A irrigação é realizada por gotejamento via fita ou tubo gotejador que é estendido no solo ao longo da fileira de plantas.

O uso de híbrido e de gotejamento é efetivo, porém é mais oneroso do que o uso de cultivar e irrigação por mangueira e influenciam marcadamente o custo de produção. Acredita-se que as duas técnicas, híbrido e de gotejamento, podem ser combinadas resultando em menor gasto. Para tal, é necessário engenhar diferentes combinações de estande, modo de desbrotar, tutorar e de distribuir a fita gotejadora no campo.

Assim, foi idealizada a técnica denominada DFT, sigla do Departamento de Fitotecnia, da UFV, a qual está detalhada no material e métodos. DFT é o oposto do sistema mencionado por alguns autores (FONTES et al., 1987; SANDRI et al., 2002) em que alta população é combinada com reduzido número de cachos por tomateiro ou com apenas um cacho por planta (LOGENDRA et al., 2001). Em DFT foram combinadas baixa densidade de plantio (D), reduzido comprimento de fita gotejadora (F) e alto número de caules por planta, especialmente tutorados (T) com fitilho plástico. Com o presente trabalho, objetivouse avaliar a técnica denominada DFT sobre a produção de frutos do tomateiro em ambiente protegido.

\section{MATERIAL E MÉTODOS}

Foram conduzidos dois experimentos em área experimental da Universidade Federal de Viçosa (UFV) em solo sob proteção de plástico de $0,1 \mathrm{~mm}$ de espessura, em estrutura rústica, na forma de capela, com dimensões de $8 \times 40 \mathrm{~m}$ e altura de $3,2 \mathrm{~m}$, com laterais possuindo cortinas possíveis de serem abertas e fechadas.

O primeiro experimento (Exp.1) foi realizado em época de temperatura amena durante a fase de colheita dos frutos (transplante em 30/01/03 e última colheita em 27/06/03) e ciclo de 148 dias após o transplante (DAT) e o segundo (Exp.2) foi realizado em época de temperatura alta (transplante em 02/10/03, primeira colheita em 22/12 e última colheita em 09/02/04) com o ciclo de 130 DAT, pouco antecipado devido à época e ao ataque de traça.

Em ambos os experimentos foram estudados dois tratamentos sendo o primeiro denominado de convencional ou testemunha (TEST), com plantio feito no espaçamento de $1,0 \mathrm{~m}$ entre fileiras e $0,6 \mathrm{~m}$ entre plantas ( 16.667 plantas ha $\left.\mathrm{h}^{-2}\right)$; cada planta foi desbrotada para 2 caules que foram tutorados verticalmente com fitilho de plástico. A planta ficou com 8 cachos, isto é, 13,3 cachos $\mathrm{m}^{-2}$. Os tutores e a fita gotejadora ficaram no sentido da fileira de plantio (FONTES \& SILVA, 2002). O segundo tratamento foi o método proposto, denominado de DFT, sigla do Departamento de Fitotecnia da UFV, oriunda das letras negritadas abaixo. Neste tratamento, o plantio foi feito no espaçamento de $2,0 \mathrm{~m}$ entre fileiras e 1,2 $\mathrm{m}$ entre plantas, densidade (D) de 4.167 plantas ha ${ }^{-1}$ sendo cada planta desbrotada para oito caules que foram tutorados (T) inclinadamente com fitilho de plástico. Foram deixados os oito primeiros caules que surgiram na planta. Um fitilho foi enrolado na base de cada caule e estendido inclinadamente em relação ao solo e preso em bambu suspenso horizontalmente ao chão, a 1,80 m de altura, formando um arranjo tipo leque na direção do meio da fileira de plantas. Completando, a fita (F) gotejadora foi estendida longitudinalmente à fileira e transversalmente ao bambu. Cada planta ficou com 32 cachos, isto é, 13,3 cachos $\mathrm{m}^{-2}$.

Os tratamentos foram instalados no delineamento inteiramente casualizado com 12 repetições nos tratamentos TEST e 6 para o DFT. Foi utilizado o híbrido Carmen. Mudas de 30 dias foram transplantadas em solo arado, gradeado, adubado e sulcado. Durante os experimentos foram realizadas capina, irrigação por gotejamento, fertirrigação com N e K, controle fitossanitário e desbrotas sempre que necessários.

No primeiro experimento, a adubação de plantio expressa por ha, foi: $570 \mathrm{~kg}$ de superfosfato simples, 100 $\mathrm{kg}$ de sulfato de magnésio, $10 \mathrm{~kg}$ de acido bórico, $10 \mathrm{~kg}$ sulfato de zinco e $0,2 \mathrm{~kg}$ de molibdato de sódio. $\mathrm{O}$ nitrocálcio e o cloreto de potássio foram parcelados, aplicando-se $10 \%$ (30 e $58 \mathrm{~kg} \mathrm{ha}^{-1}$, respectivamente) das quantidades totais antes do transplante; as quantidades restantes (270 e $522 \mathrm{~kg} \mathrm{ha}^{-1}$, respectivamente) foram fornecidas em fertirrigação, em doses iguais, no total de 12, semanalmente, a partir da segunda semana após o transplante. 
A irrigação foi por gotejamento, realizada diariamente, utilizando-se fita gotejadora com gotejadores espaçados 0,6 e 1,2 m para os tratamentos TEST e DFT, respectivamente. A necessidade de água da cultura no tratamento TEST foi determinada por lisímetro com lençol freático de nível constante para a medida da evaporação (Et $)$. A quantidade de água aplicada no tratamento DFT foi realizada três vezes ao dia. A última colheita foi feita aos 148 dias após transplante (DAT) quando não havia frutos com potencialidade de se transformarem em "frutos comerciais". Cada caule foi despontado após atingir 1,90 m.

No segundo experimento, cada caule foi despontado após a emissão de quatro cachos. A adubação de plantio, por ha, foi: $240 \mathrm{~kg}$ de sulfato de amônio, $1530 \mathrm{~kg}$ de super fosfato simples, $20 \mathrm{~kg}$ de cloreto de potássio, $300 \mathrm{~kg}$ de sulfato de magnésio, $10 \mathrm{~kg}$ de bórax e $1 \mathrm{~kg}$ de molibdato de amônio. Essas quantidades foram distribuídas e incorporadas no sulco de transplante. Nesse experimento, adicionalmente à adubação de plantio foram feitas dez aplicações de adubo em cobertura, via água de irrigação por gotejamento.

As fertirrigações iniciaram-se logo após o aparecimento do primeiro fruto e foram realizadas a cada semana totalizando 2000 e $1150 \mathrm{~kg} \mathrm{ha}^{-1}$ de nitrocálcio e cloreto de potássio, respectivamente. A solução contendo nitrocálcio era sempre preparada no dia anterior, utilizandose apenas o sobrenadante. Nos dois tratamentos foi colocada a mesma quantidade de adubo por área. A quantidade de água foi estimada pelo método do minievaporímetro (ARAÚJO et al., 2003).

Nos dois experimentos, foram anotados os números de: folha, cacho, fruto comercial e não comercial além das massas frescas e secas de folha e de caule no final do experimento. Os frutos foram colhidos totalmente vermelhos e separados em comerciais e não comerciais (< $60 \mathrm{~g}$ e com defeitos causados, principalmente por danos de insetos) e pesados obtendo-se a produção total por planta. Além da produtividade por área e por planta, foi calculada a produção por Real (R\$) gasto com os principais insumos considerando que os gastos com semente, mourão, arame, fita gotejadora e substrato foram $\mathrm{R} \$$ $12.860,00$ e R $\$ 5.570,00 \mathrm{ha}^{-1}(1 \mathrm{US} \$=3,00 \mathrm{R} \$$ ) para TEST e DFT, respectivamente. Nos dois experimentos, as características avaliadas foram submetidas à análise de variância e as médias comparadas pela significância do teste de $\mathrm{F}$ a $5 \%$ de probabilidade.

\section{RESULTADOS E DISCUSSÃO}

Quando expressos por planta, os valores das características avaliadas foram maiores em DFT; tal tendência não foi tão marcante quando os valores foram expressos por unidade de área (Tabela 1).
Em TEST e DFT, o número total de frutos por cacho foi $4,1 \pm 0,8$ e 4,2 $\pm 0,8(\operatorname{Exp} 1)$ e $6,1 \pm 1,0$ e $5,6 \pm 0,3(\operatorname{Exp} 2)$; a massa média do fruto foi $164 \pm 53$ e $123 \pm 33 \mathrm{~g}$ (Exp1) e 148 \pm 40 e $120 \pm 39 \mathrm{~g}$ (Exp2). Nos frutos ficou a maior parte do peso da matéria fresca da planta, 69 e $75 \%$ em TEST e DFT, na média dos dois experimentos, respectivamente; os valores correspondentes nas folhas foram 21 e $16 \%$ e no caule foram 10 e $9 \%$, respectivamente. Fayad et al. (2001) também mostraram que o fruto foi o principal dreno no tomateiro, acumulando mais matéria seca que folha, caule e cacho.

Na média dos experimentos, a massa de cada folha foi 47 e $22 \mathrm{~g}$ em TEST e DFT, respectivamente. Folhas menores podem indicar deficiência nutricional, especialmente de nitrogênio. Scholberg et al. (2000) mostraram que o tomateiro com deficiência de $\mathrm{N}$ produz menos e menores folhas que aquele adequadamente nutrido, resultando em menores número, diâmetro e produção de frutos (FERREIRA, 2001; GUIMARÃES, 1998). No presente trabalho, em DFT e na TEST havia 0,4167 e 1,6667 sistema radicular $\mathrm{m}^{-2}$ respectivamente. Talvez, o sistema radicular do tomateiro em DFT não tenha sido capaz de absorver todo o fertilizante disponível ou a quantidade adicionada do fertilizante não tenha sido suficiente. $\mathrm{O}$ mesmo raciocínio pode ser feito para a água.

A produtividade de fruto comercial (PFC) dependeu do modo de expressar essa variável (Tabela 2). Ao se expressar a PFC em $\mathrm{kg} \mathrm{m}^{-2}$, o tratamento TEST proporcionou melhor resultado do que o DFT, principalmente no Exp2 (Tabela 2). O inverso ocorreu tanto ao expressar a PFC em kg planta ${ }^{-1}$ quanto ao expressar em $\mathrm{kg} \mathrm{R} \$^{-1}$ (Tabela 2). Portanto, uma planta teve o potencial de produzir até 17,54 $\pm 2,08 \mathrm{~kg}$ de frutos comerciais, em ciclo de 128 dias. Expressar a produtividade em $\mathrm{kg} \mathrm{R} \${ }^{-1}$ pode ser considerado uma medida do risco do capital investido em insumos com a cultura do tomateiro.

No Experimento 1, a produtividade de fruto comercial do tomateiro (PFC) por área e por dia de permanência da cultura no campo foi 434 e $336 \mathrm{~kg} \mathrm{ha}^{-1}$ $\mathrm{dia}^{-1}$ nos tratamentos TEST e DFT, respectivamente; os valores correspondentes no Exp. 2 foram 882 e $562 \mathrm{~kg}$ $\mathrm{ha}^{-1} \mathrm{dia}^{-1}$. Valores de 682 a $1202 \mathrm{~kg} \mathrm{ha}^{-1} \mathrm{dia}^{-1}$ são possíveis de serem estimados em diversos trabalhos (FONTES, 1997; FONTES et al., 2004; PAPADOPOULOS \& HAO, 1997; VOOREN et al., 1986). Diversos fatores são responsáveis pela diferença de produtividade diária por área, sendo a população de plantas um dos mais importantes. 
TABELA 1 - Características do tomateiro nos tratamentos TEST e DFT, nos dois experimentos.

\begin{tabular}{|c|c|c|c|c|}
\hline \multirow{3}{*}{$\begin{array}{c}\text { Características } \\
\text { do tomateiro }\end{array}$} & \multicolumn{2}{|c|}{ Experimento 1} & \multicolumn{2}{|c|}{ Experimento 2} \\
\hline & \multicolumn{4}{|c|}{ Tratamentos } \\
\hline & TEST $^{1}$ & DFT $^{2}$ & TEST & DFT \\
\hline \multicolumn{5}{|l|}{ Por planta } \\
\hline $\mathrm{N}^{\mathrm{o}}$ de folha & $43,6 \pm 6,5 B^{*}$ & $201,0 \pm 28,8 \mathrm{~A}$ & $23,3 \pm 3,7 \mathrm{~B}$ & $101,7 \pm 9,3 \mathrm{~A}$ \\
\hline $\mathrm{N}^{\mathrm{o}}$ de cacho & $7,6 \pm 1,5 \mathrm{~B}$ & $31,0 \pm 3,7 \mathrm{~A}$ & $7,8 \pm 0,6 \mathrm{~B}$ & $29,8 \pm 2,1 \mathrm{~A}$ \\
\hline $\mathrm{N}^{\circ}$ total de frutos & $30,4 \pm 4,6 \mathrm{~B}$ & $129,0 \pm 30,8 \mathrm{~A}$ & $47,5 \pm 5,6 \mathrm{~B}$ & $116,2 \pm 6,2 \mathrm{~A}$ \\
\hline $\mathrm{N}^{\mathrm{o}}$ de fruto comercial & $23,4 \pm 5,4 \mathrm{~B}$ & $98,0 \pm 22,2 \mathrm{~A}$ & $46,3 \pm 5,1 \mathrm{~B}$ & $142,3 \pm 12,7 \mathrm{~A}$ \\
\hline $\mathrm{N}^{\mathrm{o}}$ de fruto não comercial & $6,9 \pm 3,6 \mathrm{~B}$ & $31,0 \pm 13,1 \mathrm{~A}$ & $1,3 \pm 1,0 \mathrm{~B}$ & $23,8 \pm 15,0 \mathrm{~A}$ \\
\hline Massa fresca de folha $(\mathrm{g})$ & $1775 \pm 399 \mathrm{~B}$ & $2969 \pm 524 \mathrm{~A}$ & $1233 \pm 22 \mathrm{~B} 3$ & $2963 \pm 628 \mathrm{~A}$ \\
\hline Massa fresca de caule (g) & $755 \pm 164 \mathrm{~B}$ & $1580 \pm 160 \mathrm{~A}$ & $803 \pm 101 \mathrm{~B}$ & $1957 \pm 301 \mathrm{~A}$ \\
\hline \multicolumn{5}{|c|}{ Por unidade de area $\left(\mathrm{m}^{2}\right)$} \\
\hline $\mathrm{N}^{\mathrm{o}}$ de folha & $72,6 \pm 10,9 \mathrm{~B}$ & $83,9 \pm 12,0 \mathrm{~A}$ & $38,6 \pm 6,2 \mathrm{~A}$ & $42,4 \pm 3,9 \mathrm{~A}$ \\
\hline $\mathrm{N}^{\mathrm{o}}$ de cacho & $12,6 \pm 2,5 \mathrm{~A}$ & $12,9 \pm 1,5 \mathrm{~A}$ & $13,1 \pm 1,0 \mathrm{~A}$ & $12,4 \pm 0,9 \mathrm{~A}$ \\
\hline $\mathrm{N}^{\mathrm{o}}$ total de frutos & $50,6 \pm 7,6 \mathrm{~A}$ & $53,7 \pm 12,8 \mathrm{~A}$ & $79,2 \pm 9,3 \mathrm{~A}$ & $69,2 \pm 2,6 \mathrm{~B}$ \\
\hline $\mathrm{N}^{\circ}$ de fruto comercial & $39,1 \pm 9,0 \mathrm{~A}$ & $40,8 \pm 9,2 \mathrm{~A}$ & $77,1 \pm 8,5 \mathrm{~A}$ & $59,3 \pm 5,3 \mathrm{~B}$ \\
\hline $\mathrm{N}^{\mathrm{o}}$ de fruto não comercial & $11,6 \pm 6,1 \mathrm{~A}$ & $12,8 \pm 5,5 \mathrm{~A}$ & $2,1 \pm 1,6 \mathrm{~B}$ & $9,9 \pm 6,6 \mathrm{~A}$ \\
\hline Massa. fresca de folha $(\mathrm{g})$ & $2925 \pm 666 \mathrm{~A}$ & $1237 \pm 218 B$ & $2055 \pm 1234 \mathrm{~A}$ & $1234 \pm 262 B$ \\
\hline Massa fresca de caule $(\mathrm{g})$ & $1258 \pm 274 \mathrm{~A}$ & $658 \pm 67 \mathrm{~B}$ & $1339 \pm 169 \mathrm{~A}$ & $816 \pm 125 \mathrm{~B}$ \\
\hline
\end{tabular}

*Em cada experimento, médias da mesma variável, seguida de mesma letra, não diferem entre si pelo teste de $\mathrm{F}$ a $5 \%$.

${ }^{1}$ TEST: testemunha, onde o plantio foi realizado no espaçamento de $1 \mathrm{~m}$ entre fileiras e $0,6 \mathrm{~m}$ entre plantas; cada planta foi desbrotada para 2 caules, tutorados verticalmente com fitilho; a planta ficou com 8 cachos; o tutoramento e a fita gotejadora ficaram no sentido da fileira de plantio.

${ }^{2}$ DFT: sistema proposto onde a densidade (D) foi 4.167 plantas ha ${ }^{-1} ; 2$ m entre fileiras e 1,2 m entre plantas; 8 caules planta $^{-1}$, tutorados (T) por fitilho de plástico, inclinado, preso em bambu suspenso a 1,80 $\mathrm{m}$ do chão, formando um arranjo tipo leque na direção do meio da fileira; a planta ficou com 32 cachos; a fita (F) gotejadora foi estendida longitudinalmente à fileira.

TABELA 2 - Produtividade de fruto de tomate comercial expressa por diferentes modos, nos tratamentos TEST e DFT, nos dois experimentos.

\begin{tabular}{lcccc}
\hline \multirow{2}{*}{$\begin{array}{c}\text { Produtividade } \\
\text { de }\end{array}$} & \multicolumn{2}{c}{ Experimento 1 } & \multicolumn{2}{c}{ Experimento 2 } \\
\cline { 2 - 5 } fruto comercial & TEST & DFT & TEST & DFT \\
\cline { 2 - 5 } & $6,42 \pm 1,55 \mathrm{~A}$ & $4,98 \pm 0,83 \mathrm{~B}$ & $11,47 \pm 1,73 \mathrm{~A}$ & $7,31 \pm 0,86 \mathrm{~A}$ \\
$\mathrm{~kg} \mathrm{~m}^{-2}$ & $3,85 \pm 9,28 \mathrm{~B}$ & $11,95 \pm 1,99 \mathrm{~A}$ & $6,88 \pm 1,04 \mathrm{~B}$ & $17,54 \pm 2,08 \mathrm{~A}$ \\
$\mathrm{~kg} \mathrm{planta}^{-1}$ & $4,99 \pm 1,20 \mathrm{~B}$ & $8,95 \pm 1,49 \mathrm{~A}$ & $8,92 \pm 1,35 \mathrm{~B}$ & $13,13 \pm 1,55 \mathrm{~A}$ \\
$\mathrm{~kg} \mathrm{R}^{-1}$ & &
\end{tabular}

*Em cada experimento, médias da mesma variável, seguida de mesma letra, não diferem entre si pelo teste de $\mathrm{F}$ a $5 \%$.

${ }^{1}$ TEST: testemunha, onde o plantio foi realizado no espaçamento de $1 \mathrm{~m}$ entre fileiras e $0,6 \mathrm{~m}$ entre plantas; cada planta foi desbrotada para 2 caules, tutorados verticalmente com fitilho; a planta ficou com 8 cachos; o tutoramento e a fita gotejadora ficaram no sentido da fileira de plantio.

${ }^{2}$ DFT: sistema proposto onde a densidade (D) foi 4.167 plantas ha ${ }^{-1} ; 2$ m entre fileiras e 1,2 m entre plantas; 8 caules planta $^{-1}$, tutorados (T) por fitilho de plástico, inclinado, preso em bambu suspenso a 1,80 $\mathrm{m}$ do chão, formando um arranjo tipo leque na direção do meio da fileira; a planta ficou com 32 cachos; a fita $(F)$ gotejadora foi estendida longitudinalmente à fileira. 
No Exp. 1, nos tratamentos TEST e DFT, a PFC foi 84,0 e $83,7 \%$ da produção total, respectivamente; os valores correspondentes no Exp. 2 foram 98,0 e 89,0 \%. Em estufa, durante os meses quente do ano, a produção de tomate é limitada principalmente pela absorção de água (BERTIN et al., 2000). Neste período, a redução da carga de frutos na planta, visando aumentar o tamanho dos mesmos, provoca o aumento da incidência de podridão apical e rachadura. Por outro lado, aumentando-se o suprimento de água, em plantas com baixa carga de frutos, há diluição da massa seca e de açúcares no fruto e, geralmente, torna o problema de rachadura mais acentuado devido ao intenso crescimento dos frutos. Isso não foi enfaticamente notado entre os tratamentos do presente trabalho.

Com grande carregamento de frutos, sem o correspondente aumento na área foliar, o fornecimento de carboidratos pode ser limitante. A disponibilidade de água e de nutrientes também pode ser limitante se o crescimento das raízes não aumentar ou se for reduzido devido a aumentada competição entre os órgãos reprodutivo e vegetativo do tomateiro pela reduzida prioridade das raízes pela participação dos assimilados (HURD et al., 1979). No presente experimento, a relação área foliar: fruto, o potencial água no solo, o desenvolvimento de raízes e a disponibilidade de nutrientes não foram medidas para confirmar a afirmativa.

O número desejado de caules por tomateiro é controlado pela poda da gema axilar. Essa prática remove fonte e dreno e afeta a produção de frutos (OLIVEIRA et al., 1995). No presente estudo, o número de caules foi igual nos dois tratamentos, 3,333 caules $\mathrm{m}^{-2}$. Adequada combinação de densidade de plantio com número de caules por planta otimiza a produtividade ou torna adequado o tamanho dos frutos às exigências do mercado (FONTES \& SILVA, 2002). A densidade de plantio e o número de caules por planta determinam a interceptação da radiação solar. Ambos determinam a área foliar disponível (fonte) por área de terreno plantado ou índice de área foliar (IAF). O IAF condiciona a interceptação da radiação solar cuja otimização é fundamental para maximizar a produção de cachos e de frutos (dreno) os quais determinam a produtividade da cultura do tomateiro. A combinação ótima depende de diversos fatores entre os quais práticas culturais, condições ambientais, preço da semente, cultivar e classe ou calibre do fruto desejado.

A discrepância dos valores verificada entre os experimentos pode ser devida à variação ambiental entre épocas de cultivo que tem grande efeito sobre a produção de tomate (WARNER et al., 2002). A maior variação entre os tratamentos ocorrida no Exp. 2 pode ter sido devido ao ataque de traça no final do ciclo, mais intensamente nas plantas no DFT que estavam pouco menos desenvolvidas do que as plantas em TEST. No Exp. 2, não houve colheita de nenhum fruto em 3 e $13 \%$ dos cachos do tomateiro nos tratamentos TEST e DFT, respectivamente; tal fato não ocorreu no Exp.1.

Há imperfeições no método DFT. As mais significativas são: impossibilidade de mecanização da lavoura caso a fita gotejadora não seja enterrada; perda de uma planta representar um problema acentuado; imperfeição no conhecimento sobre o manejo de água e fertilizante; necessidade de maior área para a obtenção da mesma produtividade de frutos; pequeno alongamento do ciclo; desconhecimento do comportamento do método em cultura no campo sem proteção. Adequações na combinação de baixa densidade de plantio com elevado número de caules por planta e maior número de cachos em cada caule poderão melhorar o desempenho do método DFT. Essas adequações necessitam ser acompanhadas do conhecimento sobre o sistema radicular, objetivandose aumentar a eficiência do manejo da água e dos fertilizantes. Também, é necessário que não ocorram problemas no final do ciclo que impeçam adequada produção de frutos nos últimos cachos. Mesmo considerando que ajustes são necessários e que há menor produtividade por área podendo ocorrer menor lucro por área, dependendo do preço de venda do fruto, o método DFT pode ser utilizado. Esse método proporciona, em média, $63 \%$ mais produção de tomate comercial por real gasto do que a testemunha, portanto oferece menor risco do capital investido em insumos.

\section{CONCLUSÃO}

O método DFT proposto pode ser utilizado na produção de tomate longa vida em ambiente protegido, pois proporciona maior produção de fruto comercial por real gasto do que o método tradicional.

\section{REFERÊNCIAS BIBLIOGRÁFICAS}

ARAÚJO, C.; FONTES, P. C. R.; RIBEIRO, J. M. O. Utilização de minievaporímetro para a determinação da quantidade de água a ser adicionada por gotejamento ao tomateiro cultivado em ambiente protegido. Horticultura Brasileira, Brasília, v. 21, n. 2, p. 286, 2003. Suplemento 1. Disponível em: <www.horticiencia.com.br/anais>. Acesso em: 10 maio 2004. 
BERTIN, N.; GUICHARD, S.; LEONARDI, C.; LONGUENESSE, J. J.; LANGLOIS, D.; NAVEZ, B. Seasonal evolution of the quality of fresh glasshouse tomatoes under mediterranean conditions, as affected by air vapour pressure deficit and plant fruit load. Annals of Botany, London, v. 85, p. 741-750, 2000.

CAMARGOS, M. I. de. Produção e qualidade de tomate longa vida em estufa, em função do espaçamento e do numero de cachos por planta. 1998. 68 f. Dissertação (Mestrado) - Universidade Federal de Viçosa, Viçosa, 1998.

CAMARGOS, M. I. de; FONTES, P. C. R.; CARDOSO, A. A.; CARNICELLI, J. H. A. Produção de tomate longa vida em estufa, influenciada por espaçamento e número de cachos por planta. In: CONGRESSO BRASILEIRO DE OLERICULTURA, 40., 2002, São Pedro. Horticultura Brasileira, Brasília, v. 18, p. 563-564, 2002. Suplemento.

CAMPOS, J. P.; BELFORT, C. C.; GALVÃO, J. D.; FONTES, P. C. R. Efeito da poda da haste e da população de plantas sobre a produção do tomateiro. Revista Ceres, Viçosa, v. 34, n. 192, p. 198-208, 1987.

CRUZ-CARRILLO, J.; JIMENEZ, F.; DIAZ, G.; SANCHEZ, P.; PERALES, C.; ARELLANES, A. Evaluacion de densidades de siembra en tomate (Lycopersicon esculentum Mill) en invernadero. Agronomia Mesoamericana, [S.1.], v. 14, n. 1, p. 85-88, 2003.

FAYAD, J. A.; FONTES, P. C. R.; CARDOSO, A. A.; FINGER, F. L.; FERREIRA, F. A. Crescimento e produção do tomateiro cultivado em condições de campo e de estufa. Horticultura Brasileira, Brasília, v. 19, n. 3, p. 365-370, 2001.

FERREIRA, M. M. M. Índices de nitrogênio para o diagnóstico do estado nutricional do tomateiro em presença e ausência de adubação orgânica. 2001. 145 f. Tese (Doutorado) - Universidade Federal de Viçosa, Viçosa, 2001.

FONTES, P. C. R. Produtividade do tomateiro: $\mathrm{kg} / \mathrm{ha}$ ou $\mathrm{kg} /$ ha/dia? Horticultura Brasileira, Brasília, v. 15, n. 2, p. 8384, 1997.

FONTES, P. C. R.; LOURES, J. L.; GALVÃO, J. C. C.; CARDOSO, A. A.; MANTOVANI, E. C. Produção e qualidade do tomate produzido em substrato, no campo e em ambiente protegido. Horticultura Brasileira, Brasília, v. 22, n. 3, 2004.
FONTES, P. C. R.; NAZAR, R.; CAMPOS, J. Produção e rentabilidade da cultura do tomateiro afetadas pela fertilização e pelo sistema de condução. Revista Ceres, Viçosa, v. 34, n. 194, p. 355-365, 1987.

FONTES, P. C. R.; SILVA, D. J. H. Produção de tomate de mesa. Viçosa: Aprenda Fácil, 2002. 196 p.

GARY, C.; BALDET, P.; BERTIN, N.; DEVAUX, C.; TCHAMITCHIAN, M.; RAYMOND, P. Time-course of tomato whole-plant respiration and fruit and stem growth during prolonged darkness in relation to carbohydrate reserves. Annals of Botany, London, v. 91, p. 429-438, 2003.

GAUTIER, H.; GUICHARD, S.; TCHAMITCHIAN, M. Modulation of competition between fruits and leaves by flower pruning and water fogging, and consequences on tomato leaf and fruit growth. Annals of Botany, London, v. 88, p. $645-652,2001$.

GUIMARÃES, T. G. Nitrogênio no solo e na planta, teor de clorofila e produção do tomateiro, no campo e na estufa, influenciados por doses de nitrogênio. 1998. 184 f. Tese (Doutorado) - Universidade Federal de Viçosa, Viçosa, 1998.

HEUVELINK, E. Dry matter partitioning in tomato: validation of a dynamic simulation model. Annals of Botany, London, v. 77, p. 71-80, 1996.

HURD, R. G.; GAY, A. P.; MOUNTFIELD, A. C. The effect of partial flower removal on the relation between root, shoot and fruit growth in indeterminate tomato. Annals of Applied Biology, Warwick, v. 9, p. 77-89, 1979.

LOGENDRA, L. S.; GIANFAGNA, T. J.; SPECCA, D. R.; JANES, H. W. Greenhouse tomato limited cluster production systems: crop management practices affect yield. HortScience, Alexandria, v. 36, n. 5, p. 893-896, 2001.

OLIVEIRA, V. R.; CAMPOS, J. P.; FONTES, P. C. R.; REIS, F. P. Efeito do número de hastes por planta e poda apical na produção classificada de frutos de tomateiro. Ciência e Prática, Lavras, v. 19, n. 4, p. 414-419, 1995.

PAPADOPOULOS, A. P.; HAO, X. Effects of three greenhouse cover materials on tomato growth, productivity, and energy use. Scientia Horticulturae, Amsterdam, v. 69, p. 1-29, 1997. 
SANDRI, M. A.; ANDRIOLO, J. L.; WITTER, M.; TIAGODAL-ROSS, T. D. High density of defoliated tomato plants in protected cultivation and its effects on development of trusses and fruits. Horticultura Brasileira, Brasília, v. 20, n. 3, p. 485-489, 2002.

SCHOLBERG, J.; McNEAL, B. L.; JONES, J. W.; BOOTE, K. J.; STANLEY, C. D.; OBREZA, T. A. Growth and canopy characteristics of field-grown tomato. Agronomy Journal, Madison, v. 92, p. 152-159, 2000.
VOOREN, J.; WELLES, G. W. H.; HAYMAN, G. Glasshouse crop production. In: ATHERTON, J. G.; RUDICH, J. (Eds.). The tomato crop: a scientific basis for improvement. London: Chapman and Hall, 1986. p. 581-623.

WARNER, J.; HAO, X.; ZHANG, T. Q. Effects of row arrangement and plant density on yield and quality of early, small-vined processin tomatoes. Canadian Journal Plant Science, Ottawa, v. 82, p. 765-770, 2002. 